\title{
La atenuación en interacciones controvertidas en comentarios sobre noticias digitales y en Twitter
}

MITIGATION IN ONLINE CONTROVERSIES IN COMMENTS ON DIGITAL NEWS AND ON TWITTER

\author{
Laetitia Aulit \\ Université catholique de Louvain
}

Resumen

Esta investigación presenta un análisis de la atenuación en un corpus de comentarios a artículos de prensa digitales y de contribuciones en Twitter sobre la fiesta de Moros y Cristianos, una tradición objeto de ciertas controversias en el debate público español. Nos basamos en Albelda y Briz (2020) quienes consideran la atenuación como una estrategia retórico-pragmática originada por necesidades de protección de la imagen y que permite al hablante lograr más eficazmente sus metas conversacionales. Por eso, este artículo se interesa no sólo por los procedimientos lingüísticos utilizados para atenuar lo dicho, sino también por los contextos interaccionales específicos en los que aparecen y las funciones que desempeñan en relación con las imágenes. Con tal propósito, esta investigación se basa en los trabajos anteriores sobre la atenuación y la metodología de análisis desarrollada por Albelda et alii (2014), adaptada a las características situacionales de las interacciones en línea objeto del estudio. Los resultados muestran que las atenuaciones aparecen principalmente con función preventiva en contextos interaccionales de desacuerdo o crítica hacia un interlocutor identificado, pero también con mucha frecuencia con función autoprotectora en intervenciones reactivas al conjunto de la discusión.

PALABRAS CLAVE: atenuación, interacciones en línea, controversia, pragmática
Abstract

This article presents an analysis of mitigation in a corpus of comments to digital press articles and contributions on Twitter about the Moors and Christians festival, a tradition that led to controversies in the Spanish public debate. Based on Albelda and Briz (2020), we define mitigation as a rhetorical and pragmatic strategy that proceeds from the need to protect the participants' face and that allows the speaker to achieve more efficiently his conversational goals. For this reason, this paper not only looks into the linguistic strategies used to mitigate, but also into the specific interactional contexts in which they appear and the functions they carry out regarding face. To that end, this investigation is based on previous research on mitigation and on the methodology developed by Albelda and al. (2014), adapted to the situational features of the online interactions studied in this research. The results show that mitigation appears mainly with preventive function in interactional contexts of disagreement or attack to an identified interlocutor, but also frequently as self-protection in responding moves to the entire discussion.

KEY WORDS: mitigation, online interactions, controversy, pragmatics 


\section{INTRODUCCIÓN}

Todo acto comunicativo entraña la implicación de la imagen social de sus participantes. La imagen social, definida por Goffman (1967) como una imagen de sí mismo delineada en términos de atributos sociales aprobados, manifiesta cómo un hablante se percibe a sí mismo y quiere que los demás le perciban en el transcurro de la comunicación. Esa imagen reivindicada por el hablante no es fija, sino que se moldea a lo largo de la comunicación dando lugar a diferentes actividades de imagen.

En el ámbito del español, prosperaron en los últimos años investigaciones sobre (des)cortesía y actividades de imagen en interacciones en línea (Mancera Rueda, 2015). Sin embargo, pocos estudios se centraron en las estrategias lingüísticas empleadas para realizar dichas actividades. Por eso, en el presente estudio se pretende analizar estrategias lingüísticas que participan en las actividades de imagen en interacciones en línea en español.

En este contexto, la atenuación ha sido considerada como un modo de expresión de la cortesía, dado que permite mitigar y reparar actos de habla perjudiciales que podrían dañar la imagen del interlocutor (Briz, 2012). No obstante, no se reduce a fenómenos de cortesía, sino que también se relaciona con la actividad argumentativa y la eficacia de la comunicación permitiendo al hablante minimizar o reducir su responsabilidad hacia lo dicho para lograr mayor aceptación por parte del interlocutor (Briz, 2005, 2012). Objeto de varias investigaciones centradas en estrategias lingüísticas específicas (Villalba 2017; Kotwica 2017, entre otros) y principalmente estudiada en géneros discursivos orales (grupo de investigación Val.Es.Co; De Cock, Marsily, Pizarro Pedraza y Rasson, 2018; entre otros), la atenuación también recibió una atención creciente en géneros discursivos digitales, entre los cuales las redes sociales (González García y García Ramón, 2017; Noblía, 2018) y los foros de comentarios a noticias de prensa (Pano Alamán, 2018 sobre temas políticos; Moya-Muñoz y Carrió-Pastor, 2018 para un estudio comparativo sobre temas políticos y deportivos).

Sin embargo, un estudio contrastivo de la atenuación en estos géneros digitales sobre temas controvertidos ajenos a la política y los deportes escasea, más aún sobre el tema de las tradiciones. Por eso, el objetivo de este artículo es presentar un análisis de la atenuación en un corpus de comentarios a artículos de prensa digitales y contribuciones en Twitter sobre una tradición celebrada principalmente en la Comunidad Valenciana y algunas localidades del sureste de España, la fiesta de Moros y Cristianos. Esta celebración conmemora las batallas de la Reconquista de la península ibérica y ha sido objeto de controversia en el debate público español. Por un lado, ha recibido algunas críticas por la representación que se da de los musulmanes, al presentarles siempre como perdedores de la lucha contra los cristianos y al celebrar actos ofensivos hacia algunos de sus símbolos religiosos en ciertas localidades. Por otro lado, también ha sido blanco de debate por la participación de las mujeres en igualdad de condiciones en desfiles y papeles tradicionalmente solo accesibles a los hombres. 
Aakhus y Lewinski (2017) defienden que las controversias contemporáneas son polílogos más que simples diálogos y se caracterizan por desarrollarse en una variedad de lugares involucrando a varios actores con una diversidad de posturas. Consideramos las interacciones estudiadas en esta investigación como reflejo y parte de tales polílogos o de debates más amplios en la sociedad acerca del tratamiento y la imagen de ciertos grupos sociales, los musulmanes y las mujeres. La elección de este tema permitirá investigar un asunto menos estudiado desde el punto de vista del análisis del discurso contribuyendo a la mejor comprensión de debates vigentes en la sociedad.

Así, el objetivo de esta investigación es analizar algunas tácticas lingüísticas que participan en las actividades de imagen, a saber, las estrategias de atenuación, en tuits y comentarios a artículos de prensa sobre una tradición española controvertida por el tratamiento de los musulmanes y las mujeres, considerando los contextos comunicativos y los papeles que desempeñan en el desarrollo de estas interacciones. Por eso, las preguntas de investigación a las que se intentará contestar son las siguientes.

(1) ¿En qué contextos comunicativos aparecen las estrategias de atenuación en las interacciones en línea estudiadas?

(2) ¿Sobre qué temas suelen versar los actos de habla atenuados?

(3) ¿Qué actividades de imagen aparecen más en estas interacciones en línea sobre temas controvertidos?

(4) ¿Qué procedimientos lingüísticos se utilizan más para realizar dichas actividades de imagen?

Con tal propósito, se presentarán en el marco teórico (sección 2), después de una caracterización del fenómeno pragmático de la atenuación (2.1), las observaciones destacadas por los estudios sobre la atenuación en los géneros digitales que son el objeto de este artículo (2.2). A continuación, se presentarán (sección 3) los datos analizados y la metodología empleada, así como el análisis llevado a cabo (sección 4). Para finalizar, se reflexionará sobre las observaciones hechas y se formularán las conclusiones del estudio (sección 5).

\section{MARCO TEÓRICO}

\subsection{La atenuación}

En el ámbito del español, la atenuación ha sido objeto de gran interés. La atenuación fue definida como una «estrategia retórico-pragmática originada por necesidades de protección de la imagen, dirigida a mitigar los posibles efectos perjudiciales para el desarrollo adecuado de la comunicación" y "permite a los emisores formular un menor compromiso con lo dicho y así lograr más eficazmente las metas conversacionales de los participantes en el discurso" (Albelda y Briz, 2020: 582). Así, la atenuación consiste en una estrategia lingüística que se utiliza al servicio de los propósitos comunicativos del hablante, dado que permite buscar el acuerdo y la aceptación por parte del oyente (estrategia retórico-argumentativa), cuidando simultáneamente las imágenes de las personas involucradas en la comunicación (Albelda y Briz, 2020).

Como lo demuestra Schneider $(2010 ; 2017)$ a través del examen de los enfoques en torno a este fenómeno, la clasificación de los mecanismos de atenuación ha ido evolucionando 
y especificándose a lo largo de los años. A partir del concepto de hedge introducido por Lakoff (1973), Brown y Levinson (1978; 1987) añadieron bajo este término el concepto de modificación del compromiso del hablante hacia la verdad de lo dicho. A continuación, varios investigadores se basaron en estas propuestas para desarrollar su propia concepción de la atenuación. Por ejemplo, Caffi (1999), cuya clasificación fue tomada como referencia en muchas reflexiones sobre la atenuación (entre otros Albelda y Cestero, 2011, de quienes tomamos la traducción en español de las propuestas de Caffi; Albelda y Estellés, 2021), distingue tres tipos de mecanismos atenuantes: los bushes que «afectan al contenido proposicional» reduciendo su precisión o cantidad, los hedges que «inciden en la fuerza ilocutiva reduciendo el compromiso del hablante con una aseveración» (divergiendo en parte del significado atribuido por Brown y Levinson para el mismo término), y los shields que atenúan «el origen o fuente del enunciado».

Sin embargo, no existe un listado de formas específicas que siempre actúan con valor atenuante, sino que son estrategias lingüísticas que pueden, en función de su uso, producir este efecto de atenuación. A este respecto, Albelda (2010) destaca que la aparición de actos de habla atenuados en la comunicación es influenciada por la fuerza ilocutiva del acto de habla, la implicación de las imágenes de los interlocutores en la interacción, así como sus rasgos situacionales (el marco físico, el origen geográfico y cultural, y la relación de poder, proximidad y conocimientos compartidos entre los interlocutores). Por consiguiente, en función de la situación y del tipo de acto realizado, la atenuación puede aparecer para cumplir tres funciones distintas según la imagen que se ve afectada por el proceso comunicativo: la autoprotección cuando el hablante vela por su propia imagen cuidando su manera de expresarse y/o disminuyendo o evitando responsabilidades sobre lo dicho o hecho; la prevención cuando el hablante intenta prevenir un conflicto o un posible daño a la imagen del otro porque lo dicho implica o ataca su imagen, sea positiva o negativa; o la reparación cuando el hablante intenta reparar daños ya producidos en la comunicación, sea ataques a la imagen del otro, conflictos surgidos por desacuerdo o problemas en la relación interpersonal (Briz, 2012; Albelda et alii, 2014).

\subsection{La atenuación en géneros discursivos digitales}

Cada género discursivo en línea se distingue por características específicas en cuanto a la situación comunicativa, al fin de la interacción y a la relación interpersonal entre los interlocutores. Habida cuenta de la influencia de los rasgos situacionales y la implicación de la imagen de los participantes en la interacción sobre la aparición de la atenuación, nos centraremos en este artículo en dos ámbitos determinados, el foro de comentarios a artículos de prensa y las interacciones en Twitter, porque ambos permiten compartir opiniones sobre temas de actualidad y presentan características situacionales semejantes.

La introducción del presente artículo ha destacado que algunos estudios ya se han interesado por el fenómeno de la atenuación en comentarios de prensa en español. En su estudio sobre las estrategias de atenuación empleadas en actos asertivos a noticias políticas del diario El País, Pano Alamán (2018), con base en la metodología de análisis propuesta por Albelda et alii (2014), comprobó que la atenuación en este tipo de discurso aparece con propósito argumentativo y conversacional, mayoritariamente con función de 
autoprotección, esto es, para evitar o reducir el compromiso del hablante con lo dicho, especialmente cuando el tema tratado se considera delicado. Asimismo, la autora observó que la función preventiva, menos frecuente, aparece tanto para dirigirse al periodista o diario, como al conjunto de lectores o interlocutores específicos.

Por su parte, Moya Muñoz y Carrió-Pastor (2018) estudiaron la variación de la atenuación en comentarios a dos periódicos, El País en España y Emol en Chile, sobre temas políticos y deportivos. Sus observaciones les permitieron constatar que los comentaristas españoles suelen atenuar más que sus homólogos chilenos, pero no distinguieron variación significativa según la temática tratada. Asimismo, al igual que en el estudio que acaba de mencionarse de Pano Alamán (2018), Moya Muñoz y Carrió-Pastor (2018) observaron que los mecanismos de atenuación más frecuentes son los que permiten expresar duda o incertidumbre, sobre todo por parte de los locutores españoles.

En la investigación sobre Twitter, Page (2014) analizó disculpas de empresas a quejas de clientes en inglés, destacando que se suelen utilizar estrategias de atribución de la responsabilidad del problema a terceros o factores independientes, así como estrategias de atenuación en actos de pregunta de la empresa al cliente (estructuras condicionales, verbos modales y fórmulas como please) y actos de queja del cliente mismo (marcadores del discurso, emoticonos). Sin embargo, hasta ahora, aún no se ha estudiado la atenuación en interacciones sobre tradiciones polémicas y sus repercusiones en la sociedad actual llevadas a cabo en esta red social, con lo cual este artículo también pretende ofrecer primeras observaciones sobre el tema.

\section{Metodología}

El presente artículo es parte de una investigación ${ }^{1}$ sobre interacciones controvertidas en línea con relación a tres tradiciones españolas que suscitaron varios debates por el tratamiento o la imagen vehiculada de ciertos grupos sociales, con especial interés en las estrategias de atenuación e intensificación empleadas para reaccionar en este tipo de interacciones. Como se ha mencionado anteriormente, este artículo trata sobre la fiesta de Moros y Cristianos, una fiesta que suscitó varias controversias relacionadas con la imagen de los musulmanes y la participación de las mujeres.

Con ese propósito, se recopilaron artículos periodísticos procedentes de los principales diarios nacionales y regionales en versión digital a partir de palabras claves con la ayuda del Centre de Traitement Automatique du Langage de la Université catholique de Louvain (Naets, 2018). De este modo también se buscaron contribuciones en Twitter, añadiendo la búsqueda por hashtags y búsquedas manuales. Luego, se seleccionaron manualmente los datos estudiados según el periodo de publicación (desde 2010 hasta 2020 porque corresponde con el momento en el que las controversias están vivas y suscitan debates en línea), el tema del artículo o de las contribuciones en Twitter (la fiesta en sí misma y las

\footnotetext{
1 Tesis doctoral en el marco del proyecto titulado Atenuación e intensificación para el posicionamiento en situaciones de controversia: el caso de tres debates españoles en el discurso oral y en los nuevos medios financiado por el Fondo Especial de Investigación de la Université catholique de Louvain.
} 
controversias que aparecieron en la sociedad) y la presencia de comentarios en reacción al artículo periodístico o la contribución en Twitter.

Así, se escogieron todos los hilos que cumplían con los criterios, con lo cual se recolectaron 281 comentarios repartidos en respuestas a 19 artículos periodísticos distintos y 1128 intervenciones en Twitter repartidas en 27 hilos de discusión. El número de palabras analizadas en cada plataforma muestra que, si bien la fiesta y la controversia relacionada generan más reacciones en Twitter que en la prensa, los comentarios de prensa suelen ser más largos que las contribuciones en Twitter.

Tabla 1. Corpus de intervenciones analizadas

\begin{tabular}{|l|l|l|}
\hline & Prensa & Twitter \\
\hline Hilos de discusión & 19 & 27 \\
\hline Intervenciones analizadas & 281 & 1128 \\
\hline Número de palabras & 13345 & 21036 \\
\hline
\end{tabular}

La metodología de análisis se basa en las claves propuestas por Villalba (2018) y la ficha metodológica para el análisis pragmático de la atenuación desarrollada por Albelda et alii (2014). Principalmente empleada para analizar la atenuación en géneros discursivos orales, esta metodología también sirvió de punto de partida para el estudio en géneros escritos, con algunos cambios cuando fue necesario. En nuestro caso, teniendo en cuenta las características contextuales propias de las interacciones en línea aquí estudiadas, se necesitaron algunas adaptaciones presentadas a continuación (Aulit, manuscrito):

(1) Aspectos situacionales: las interacciones estudiadas se presentan como hilos de discusión, con lo cual este estudio se centra en la identificación del tipo de intervención y del fin del acto de habla. Así, el análisis del tipo de intervención permitió destacar si era iniciativa o reactiva, y siendo ese el caso si reaccionaba a la intervención de uno o varios interlocutor(es) destacables, al contenido de un artículo o enlace, o al conjunto de la discusión (en caso de no relacionarse explícitamente con otra intervención o información mencionada). Asimismo, el análisis del fin del acto de habla posibilitó identificar si el acto expresaba una postura propia del hablante sin posicionarse con respecto a otra intervención (reaccionar), si se oponía a (contradecir) o reafirmaba (apoyar) algo dicho anteriormente, o si se dirigía a alguien para criticarle o pedirle algo (dirigirse a un/varios interlocutor.es).

(2) Temática del acto de habla: puesto que este tipo de interacciones suele caracterizarse por digresiones, este estudio buscó identificar si la atenuación aparecía relacionada con el aspecto polémico de la fiesta o ciertos temas específicos. Por consiguiente, se determinó si el acto de habla versaba sobre la temática inicialmente debatida, la fiesta en sí misma, o la sociedad de forma general, o consistía en un ataque al interlocutor.

(3) Estrategias lingüísticas: por un lado, se integraron en el catálogo de estrategias lingüísticas los elementos tipográficos propios de la escritura digital que pueden actuar pragmáticamente (el uso estratégico de la puntuación y los emoticonos) porque permiten reforzar o mitigar la fuerza ilocutiva del acto de habla (Dresner y Herring, 2010; Figueras, 2014; Yus, 2014). Por otro lado, se reorganizaron las estrategias lingüísticas de atenuación propuestas por Albelda et alii (2014) en categorías establecidas en función del efecto general producido por la táctica atenuante (debilitación del contenido proposicional, por ejemplo). Asimismo, se introdujo, junto a la suavización de la fuerza ilocutiva, la identificación del tipo de acto de habla propuesta por Albelda et alii (2014) a partir de la tipología de Searle (1969) y se renombraron algunas variantes de las categorías establecidas para destacar mejor las estrategias lingüísticas que participan en cada efecto producido. 
(4) Función de la atenuación: a partir de las funciones de la atenuación destacadas por Albelda et alii (2014) y de las consideraciones sobre los tipos de públicos posibles ${ }^{2}$, se incluyó, en el análisis mismo de la función, la identificación del participante (locutor, interlocutor (no) designado o tercero) cuya imagen se veía afectada por el contenido proposicional.

Estos cambios permitieron analizar el fenómeno de la atenuación tomando en cuenta las características específicas de las interacciones en línea, como veremos en el análisis de los datos a continuación.

\section{ANÁLISIS Y RESULTADOS}

\subsection{Resultados generales}

El análisis de los datos muestra que aparecen bastante pocos fenómenos de atenuación en el tipo de interacciones objeto de esta investigación. Como se aprecia en la tabla 2, aparecen estrategias atenuantes en $13,52 \%$ de los comentarios de prensa y en 5,85\% de las intervenciones de Twitter, lo cual significa un porcentaje bastante bajo. Este resultado parece confirmar la tesis formulada en estudios anteriores que postulan una escasa presencia de atenuación en este tipo de interacciones en línea, frente a la intensificación (Pano Alamán, 2018).

Tabla 2. Resultados generales del análisis de la atenuación

\begin{tabular}{|l|l|l|}
\hline & Prensa & Twitter \\
\hline Intervenciones analizadas & 281 & 1128 \\
\hline Intervenciones con atenuación & 38 & 66 \\
\hline Actos de habla atenuados & 51 & 71 \\
\hline Porcentaje de intervenciones con atenuación & $13,52 \%$ & $5,85 \%$ \\
\hline
\end{tabular}

Sin embargo, observamos una mayor presencia de intervenciones con atenuación en el corpus de comentarios de prensa que en el corpus de intervenciones en Twitter. Tras aplicar la prueba estadística chi $^{2}$ se comprobó que hay dependencia significativa entre la presencia de atenuación en la intervención y la plataforma utilizada $(p<.0001)$.

\subsection{Contextos comunicativos de aparición de los actos de habla atenuados}

Como se ha mencionado en la parte metodológica, el análisis de los rasgos situacionales de las interacciones estudiadas se ha centrado en el tipo de intervención y su relación con respecto a las demás intervenciones del mismo hilo considerando no solo las indicaciones transmitidas por el medio (por ejemplo, en respuesta a) sino también, y, sobre todo, el contenido de lo dicho. Se destaca del gráfico 1 que las estrategias de atenuación aparecen en gran parte en intervenciones reactivas a la intervención de uno o varios interlocutor(es) específico(s) y, en menor medida, en intervenciones reactivas al conjunto de la discusión,

\footnotetext{
2 A partir de las observaciones de Goffman (1981) sobre los marcos de participación, se determinaron los públicos posibles teniendo en cuenta el tipo de intervención (iniciativa o reactiva a la intervención de otro interlocutor/ al contenido de un artículo o enlace/ al conjunto de la discusión) y el fin del acto de habla (reaccionar, contradecir, apoya, dirigirse al otro interlocutor).
} 
con una diferencia mucho más marcada en el corpus de Twitter que en el corpus de comentarios de prensa.

Gráfico 1. Tipo de intervención con atenuación

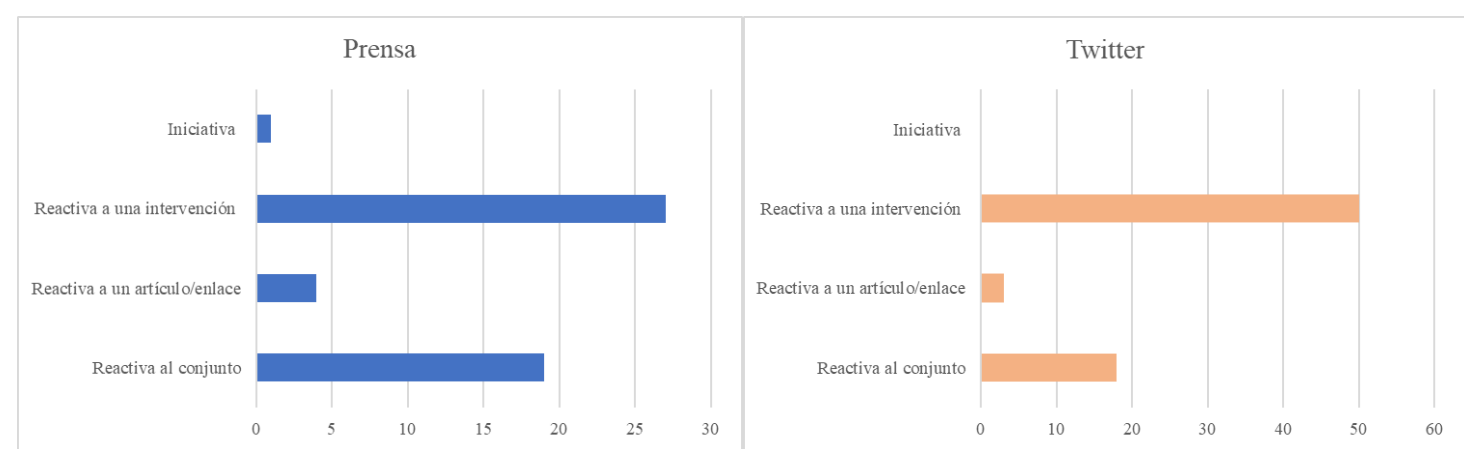

Al profundizar en el análisis del contexto interaccional, se observan tendencias distintas según el tipo de intervención. Cuando la intervención reacciona al conjunto de la discusión, el hablante emplea tácticas atenuantes mayoritariamente en actos de habla en los que expresa una postura propia sin posicionarse con respecto a los demás interlocutores. El ejemplo (1) ilustra una reacción al conjunto de una discusión cuyo punto de partida es un artículo sobre la integración de la mujer en las fiestas de Alcoy.

(1) Seudónimo1 ${ }^{3}$ 06.abr.2012 | 19:06 \#33

creo que estan haciendo un rodaje del desembarco de normandia de la segunda guerra mundial y las feministas han hecho una protesta de que en el rodaje tiene que haber 50\% de mujeres en la escena del desembargo o sino pararan el rodaje y tiene visos de que lo puedan conseguir. Tu crees que esto podria ser verdad? pues al paso que vamos.... si. [...] [ELM2012a]

En esta intervención, el hablante atenúa su primer acto de habla, una reacción que no se presenta como un apoyo, un desacuerdo o una crítica o pregunta con respecto a lo dicho anteriormente, sino como una información que transmite disminuyendo su compromiso hacia la verdad de lo dicho por medio del verbo creo que, lo cual introduce una lectura débil epistémica o de creencia que le permite expresar una duda respecto a la certeza del mensaje (Soler Bonafont, 2016).

En cambio, en las intervenciones reactivas a algo dicho por uno o varios interlocutor(es) específico(s), se suele utilizar la atenuación tanto cuando se contradice, como cuando se dirige al interlocutor para criticarle o preguntarle algo, como muestran los ejemplos a continuación.

\footnotetext{
${ }^{3}$ Reproducimos el contenido de las intervenciones tal cual sin corregir errores de lengua. Sin embargo, por motivos de privacidad, hemos elegido anonimizar los datos de cara a su tratamiento y cita, a excepción de los datos de figuras públicas. Así, se sustituyeron los nombres de usuario por "nombre" o "seudónimo", seguido de un número que permite distinguir a los interlocutores. Por motivos de claridad en la presentación de los ejemplos, se ha elegido poner en evidencia con cursivas los actos de habla atenuados y resaltar con letra negrita las estrategias lingüísticas de atenuación.
} 
(2) Turismo Alicante @Alicante_City Jul 31

El 4 de agosto Alicante se viste de \#tradición,,,el III Gran Desembarco de Moros y Cristianos inunda nuestra playa del \#Postiguet. Un evento que no os podéis perder!!! \#soleatealicante \#morosycristianos Seudónimo2 5:05 PM - Aug 1, 2019.

Hombre, que el evento no esta mal y tal. Todo lo contrario. Fue un acierto implantarlo. Con mejoras eso si. Pero de ahi a que sea una tradicion.... [TWI2019a]

(3) Seudónimo3 06.abr.2012 | 17:43 \#24

\#11 ni tú te lo crees, los "trajes" (más bien disfraces) que usan en Alcoy distan mucho de lo que fueron las vestimentas tradicionales. Las comparsas parecen cada vez más que van a un desfile de Río de Janeiro o una puesta en escena del Circo del Sol. A menos que alguien se quiera creer que durante la ocupación los musulmanes iban ataviados como figurantes de un musical de Broadway... [ELM2012a]

En (2), el hablante expresa un punto de vista personal que consiste en un desacuerdo hacia el carácter de tradición del evento, el cual atenúa mediante la partícula apelativa hombre, la formulación concesiva, la aproximación en no está mal y tal, y la elipsis de la conclusión. En cuanto al ejemplo (3), ilustra una crítica atenuada mediante el empleo de puntos suspensivos que reducen la fuerza ilocutiva, así como la impersonalización que permite evitar mencionar directamente al interlocutor, introducida por la locución conjuntiva condicional a menos que, que presenta la crítica sobreentendida como algo hipotético, lo que confirma la reflexión de Chodorowska-Pitch (2017) según la cual en las construcciones condicionales la atenuación surge del valor hipotético de la construcción.

Cabe destacar que también aparecen tácticas atenuantes en reacciones a la intervención de un interlocutor específico sin que el hablante se dirija a él o formule un desacuerdo, como en (4).

(4) Seudónimo4 10:40 AM · Apr 11, 2016

El Gobierno tripartito de Valencia podría suspender las Fiestas de Moros y Cristianos http://ow.ly/10vQUe SON GILIPOLLAS

Nombre1 Apr 11, 2016

Replying to @Seudónimo4

@Seudónimo4 y lo harán porque en este pais tragamos con todo, despotricamos en casa, nos cabreamos pero ahí acaba todo,por eso digo..... [TWI2016c]

El hablante contesta al internauta del tuit inicial expresando su punto de vista personal sobre la posible suspensión de la fiesta, que atenúa mediante una construcción justificadora del decir por eso digo. Esta construcción le permite justificar su opinión sobre la suspensión de la fiesta (lo harán) presentándola como una consecuencia de lo que observa en la sociedad española (en este país tragamos con todo, despotricamos en casa, nos cabreamos pero ahí acaba todo). De esta forma, se autoprotege limitando sus responsabilidades hacia esta postura.

En suma, el análisis demuestra que los actos de habla atenuados aparecen sobre todo en intervenciones reactivas a la intervención de otro interlocutor específico (en actos apelativos o de desacuerdo), pero también en intervenciones reactivas al conjunto de la discusión (en actos de expresión de una opinión personal independientemente de las posturas anteriores expuestas). 


\subsection{Temáticas tratadas en los actos de habla atenuados}

En lo concerniente a las temáticas de los actos atenuados, el análisis pone de manifiesto que las estrategias de atenuación aparecen tanto en actos de habla centrados en la fiesta, tal como lo ilustra el ejemplo (2) antes mencionado, como en actos de habla relativos a la sociedad de forma general, al igual que en el ejemplo (1) sobre las mujeres. Sin embargo, mientras que los comentarios de prensa analizados no manifiestan diferencia relativa a la aparición de atenuación según la temática tratada (la fiesta o sociedad), el gráfico 2 permite observar una ligera diferencia en los datos procedentes de Twitter, puesto que aparecen más actos sobre la sociedad que sobre la fiesta en sí misma.

Gráfico 2. Temática de los actos atenuados

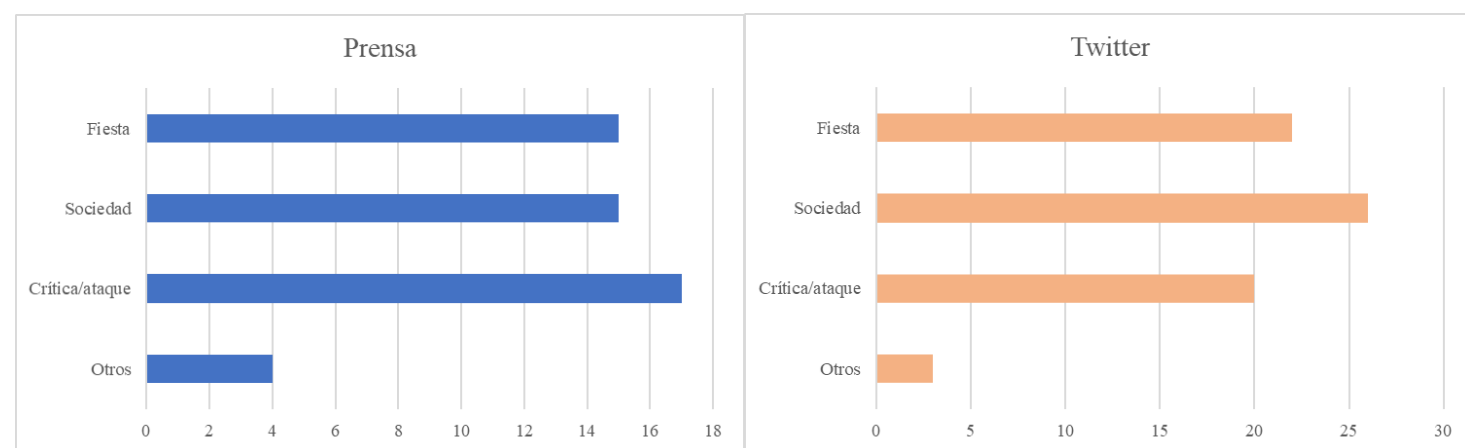

Además, se constatan con mucha frecuencia y en ambos corpus tácticas atenuantes en actos de crítica o ataque directo a la imagen. Al respecto, cabe destacar que, mientras en Twitter se atenúan más actos de habla cuyo objeto es la fiesta o la sociedad, en el corpus de comentarios de prensa se aprecia un mayor número de actos de crítica o ataque atenuados. Esta predominancia de atenuación en actos de crítica frente a actos de expresión de una postura sobre la fiesta o la sociedad podría explicarse por el componente cultural de la imagen según el cual una de las premisas culturales de la sociedad española es la tolerancia hacia la expresión de opiniones, aun si son distintas (Bravo, 1999). Así, la mayor presencia de atenuación en críticas o ataques frente a expresión de opiniones personales en el corpus de prensa podría relacionarse con el hecho de que se suela atenuar menos una expresión de opinión en la cultura española, especialmente en un género discursivo cuya principal característica es esa.

Dicho esto, en ambos corpus, observamos que el destinatario de la crítica atenuada suele ser un interlocutor internauta específico como en el siguiente ejemplo en el que el locutor formula una crítica hacia el político y su partido.

(5) Javier Ortega Smith @Ortega_Smith

1/2 Desde Alcoy (Alicante), en defensa de nuestras tradiciones, de nuestra cultura y de nuestra historia. \#Alcoy \#morosycristianos \#EspañaViva

Seudónimo5 May 6

Pues en @VOX_Valencia no lo estais haciendo muy bien.... [TVI2019b] 
(6) Seudónimo6 06.abr.2012 | 17:27 \#21

\#11 hombre, decir que se cuida el detallen la vestimenta es verdad, pero decir se cuida el rigor histórico en la vestimenta en Alcoy es no haber ido nunca a ver las fiestas. [ELM2012a]

En (5), el locutor formula una crítica hacia el político y su partido, pero la atenúa gracias a la litote (no lo estáis haciendo muy bien) y los puntos suspensivos que suavizan la fuerza ilocutiva de su acto de habla. También aparecen fenómenos de atenuación en insinuaciones que critican o atacan la imagen del otro, como en (6). En este ejemplo, el hablante expresa un desacuerdo hacia su interlocutor insinuando que no conoce las fiestas, pero atenúa lo dicho mediante la partícula apelativa hombre, el acuerdo parcial y el uso del infinitivo para impersonalizar su acto de habla y así evitar mencionar a su interlocutor.

Además, se observan unos pocos casos de atenuación de una crítica hecha al periódico o periodista y algunas críticas atenuadas hacia terceros. En el ejemplo (7), el hablante atenúa su sugerencia al periodista mediante el condicional, así como, en el segundo acto de habla atenuado, el operador modal quizás y el minimizador un poco.

(7) Seudónimo7 28.mar.2013|08:50 \#3

No sé si el trasfondo de esta noticia, como se sugiere, tiene matices sexistas o discriminatorios. Debería aclarar el periodista la postura de la Filà. [...] Si, de alguna manera, se impide a las mujeres el disfrute de la tradición, me parece mal. Si lo que se pretende es mantener la tradición en la asignación de trajes, pues no. Quizás habría que ampliar un poco la noticia. Así, es difícil opinar de forma categórica. [ELM2013a]

En conclusión, observamos que las estrategias de atenuación aparecen en ambos corpus tanto en actos de habla sobre la fiesta en sí misma como en actos sobre la sociedad de forma general, y también con cierta frecuencia en críticas dirigidas principalmente a otro interlocutor que no sea el periodista o autor del tweet inicial, lo cual destaca el carácter interaccional de estos hilos de discusión.

\subsection{Actividades de imagen realizadas mediante las estrategias de atenuación}

El análisis ha permitido destacar que las estrategias atenuantes aparecen en estas intervenciones sobre temas polémicos principalmente con función preventiva. Sin embargo, tal como demuestra la tabla 3, también aparecen frecuentemente estrategias de atenuación con función autoprotectora. Además, se observan en ambos corpus pocas diferencias en términos de frecuencia de aparición entre la función autoprotectora y la función preventiva.

Tabla 3. Función de la atenuación

\begin{tabular}{|l|l|l|}
\hline & Prensa & Twitter \\
\hline Autoprotección & 23 & 34 \\
\hline Prevención & 27 & 35 \\
\hline Reparación & 1 & 2 \\
\hline
\end{tabular}


Al considerar el tipo de intervención y el fin del acto de habla, se desprende que las estrategias atenuantes que se utilizan para autoprotegerse aparecen sobre todo en actos de habla que consisten en reacciones sin posicionarse respecto a los demás interlocutores, como en (8).

(8) Seudónimo8 13.nov.2011 | 1:49\#7

PORFAVOR! En alcoy una mujer desfilando es algo impensable ( y doy gracias) seria creo yo una deshonrra para el pueblo alcoyano.Asique no querais cambiar las tradiciones. PER ALCOI , I PER SANT JORDI [ELM2012a]

Este ejemplo ilustra un acto de habla que consiste en la expresión de una postura personal sobre la participación de la mujer en una intervención reactiva al conjunto de la discusión. La estructura creo yo, que suele utilizarse para enfatizar que se trata de una opinión personal (Soler Bonafont, 2016), le permite al hablante reducir el efecto negativo de su opinión y así autoprotegerse en un género discursivo caracterizado por la expresión de opiniones personales.

Sin embargo, algunas intervenciones en Twitter también presentan actos de habla atenuados con función autoprotectora que consisten en reacción de oposición, apoyo o crítica hacia otras intervenciones específicas. El ejemplo (9) ilustra un acto en el que el hablante se autoprotege al presentarse como experimentador de una sensación y no como responsable de lo dicho en una intervención en la que se opone a la postura de su interlocutor. Con tal propósito, suaviza el ataque que le está haciendo mediante una despersonalización que le permite atribuir la postura presentada a factores externos.

(9) Seudónimo9 Sep 22 Replying to @Seudónimo

Algo positivo? El que el enaltecimiento de la violencia?

Mejor nos iria si repasaramos los valores en que se sustentan los pilares de nuestra sociedad.

Seudónimo10 Sep 22

Ver "enaltecimiento de la violencia" en una fiesta de moros y cristianos es una lectura francamente errónea. Me da la sensación de que no has estado en ninguna. [...] [TWI2019]

Asimismo, se destaca que la función preventiva aparece sobre todo en intervenciones de oposición o reacción apelativa a algo dicho por uno o varios interlocutor(es) anterior(es), como en los ejemplos (2) y (3) antes mencionados. En (2), el hablante emplea varias tácticas atenuantes para prevenir el posible daño a la imagen de Turismo Alicante al expresar un desacuerdo hacia lo dicho, que pone en tela de juicio su postura, sin formularlo tajantemente. En (3), en cambio, la atenuación aparece en la crítica sobreentendida porque la impersonalización permite proteger la imagen del interlocutor al evitar dirigirse a él de manera abierta.

En el corpus de comentarios de prensa, se observan además algunas tácticas atenuantes con función preventiva en intervenciones reactivas al conjunto de la discusión, sea en actos que expresan una postura propia o en actos que se posicionan con respecto a lo dicho anteriormente. En (10), el hablante reacciona al conjunto de una discusión sobre una posible suspensión de la fiesta de Moros y Cristianos para no dañar a los musulmanes, y acaba su intervención dirigiéndose a sus interlocutores. El empleo de la primera persona 
del plural (dejemos) le permite incluirse en la acción y así mitigar el efecto de su exhortación porque no menciona directamente a sus interlocutores.

(10) (Nombre2 14/04/2016 12:06

Desde mi punto de vista esto es simplemente publicidad engañosa para poder poner encontra a toda la gente festera.

Yo soy festero y en mi localidad las fiestas son algo muy importante y que para mucha gente(incluyéndome) significan mucho,asi que dudo muchísimo que esta noticia sea cierta y en caso de serlo estoy segurísimo que aran un referendum en donde nos daran la opcion de elegir si o no y estoy muy seguro de que saldra que no.

Así que por amor de dios,dejemos de difamaciones inciertas y de especulaciones sin sentido.[...] [ALE2016a]

En suma, el análisis de las actividades de imagen permite resaltar dos grandes tendencias. Por un lado, la atenuación suele aparecer con función preventiva en respuestas apelativas o de desacuerdo con respecto a algo dicho por uno o varios interlocutor(es) específico(s), lo cual refuerza lo mencionado anteriormente sobre la importancia del carácter interaccional de este tipo de discusiones y del desarrollo de un polílogo. Por otro lado, también se observan estrategias atenuantes con función generalmente autoprotectora en expresión de una postura propia que reacciona al conjunto de la discusión.

\subsection{Estrategias lingüísticas de atenuación}

Como se desprende de los ejemplos mencionados hasta ahora, existe una diversidad de estrategias lingüísticas que pueden emplearse para atenuar lo dicho. En el marco de este análisis, se clasificaron las estrategias lingüísticas según el efecto general que producen basándose en las observaciones de Caffi (1999) y las estrategias destacadas por Albelda et alii (2014). Asimismo, la tabla 4 permite constatar en ambos corpus una predominancia de uso de los procedimientos que suavizan la fuerza ilocutiva del acto de habla.

Tabla 4. Efecto general de las estrategias de atenuación empleadas

\begin{tabular}{|l|l|l|}
\hline & Prensa & Twitter \\
\hline Debilitación del contenido proposicional & 7 & 7 \\
\hline Suavización de la fuerza ilocutiva & 45 & 72 \\
\hline Reducción de la fuerza ilocutiva por restricción & 4 & 4 \\
\hline Reducción de la fuerza ilocutiva por impersonalización & 9 & 15 \\
\hline Reformulación & 2 & 2 \\
\hline
\end{tabular}

Al interesarse por las estrategias lingüísticas específicamente empleadas para suavizar la fuerza ilocutiva del acto de habla, se observa que los hablantes recurren frecuentemente al uso modalizado de los tiempos verbales y a verbos con valor léxico atenuante para realizar la atenuación.

Como se ha ilustrado a partir de algunos ejemplos anteriores, se utilizan sobre todo el condicional y los verbos cognitivos con lectura modal para presentar lo dicho de forma menos hiriente y/o reducir las responsabilidades hacia lo dicho. Así, en el ejemplo (7) antes mencionado, el uso del condicional en los verbos deónticos permite presentar lo dicho, 
una crítica hacia el periodista, como una sugerencia, lo cual cumple una función preventiva que reduce el daño hecho hacia la imagen del periodista. En cuanto a (1), el verbo cognitivo creo que permite reducir la toma de posición del hablante respecto a una información que se podría comprobar, lo cual constituye un movimiento de autoprotección por parte del hablante.

La realización de una prueba estadística chi² permitió comprobar que no hay dependencia significativa entre las estrategias de atenuación de la fuerza ilocutiva y la plataforma utilizada. Sin embargo, el análisis cualitativo parece mostrar que los operadores modales y los elementos propios del código escrito digital, a saber, los emoticonos y la puntuación con función estratégica, son procedimientos que aparecen más para atenuar lo dicho en el corpus de Twitter que en el corpus de prensa, generalmente con una función preventiva hacia la imagen del interlocutor, como en los ejemplos a continuación.

(11) Seudónimo11

Podemos prohibirá la tradición de los 'Moros y Cristianos' para no ofender al Islam https://mediterraneodigital.com/portada/nacional/edicion-comunidad-valenciana/alicante/podemosprohibira-la-tradicion-de-los-moros-y-cristianos-para-no-ofender-al-islam.html

Seudónimo12 Replying to @Seudónimo11 7:18 PM · Jan 6, 2019

Igual si a alguien le da por abrir y leer la noticia se dara cuenta de que es del 2015 .. [TWI2019d]

(12) Nombre3 Jan 6, 2019

A mí y a muchos españoles nos ofende Podemos, ¿nadie lo prohibe?

Seudónimo13 Jan 6, 2019

Ofende más a la inteligencia que os tragueis estos bulos ;-) [TWI2019d]

En (11), el hablante reacciona al conjunto de la discusión empezada por Seudónimo11. Además de la impersonalización, el uso del operador modal igual y de los dos puntos suspensivos atenúa la crítica hecha hacia el comportamiento de sus interlocutores, dado que el operador modal le permite presentar lo dicho sin afirmarlo y los puntos suspensivos atenúan la fuerza ilocutiva de su aserción. Asimismo, el ejemplo (12), proveniente de la misma discusión, ilustra un acto de habla que constituye una potencial amenaza a la imagen del interlocutor al que contesta el hablante. Sin embargo, la presencia del emoticono que representa una cara haciendo un guiño atenúa la carga ofensiva de la crítica, al permitir acercarse a su interlocutor.

Finalmente, los ejemplos mencionados hasta ahora también ilustran otros efectos atenuantes presentes en menor medida en las intervenciones estudiadas. Se encuentran algunos casos de debilitación del contenido proposicional, como en los ejemplos (7) con el modificador un poco y (5) con la litote no lo estáis haciendo muy bien. Los ejemplos (3), (6) y (10) ejemplifican casos de reducción de la fuerza ilocutiva por impersonalización mediante el uso, respectivamente, de sujeto genérico (alguien), de infinitivos (decir... es no haber ido nunca), o de la primera persona del plural (dejemos de). También se encuentran unos pocos casos de atenuación por restricción de lo dicho a un determinado ámbito, personal o no, como en (8) con creo yo y reformulaciones de un acto de habla anterior. 


\section{CONCLUSIONES}

El análisis de comentarios a artículos de prensa y discusiones en Twitter sobre una fiesta tradicional debatida en la actualidad española por el tratamiento de ciertos grupos sociales permite constatar una relativa poca presencia de fenómenos de atenuación en este tipo de interacciones. Sin embargo, se necesitarían comparaciones con otros modos de interacción en línea para poder afirmar esta conclusión para la comunicación en línea de forma general. Además, este estudio contrastivo permitió observar una presencia significativamente mayor de la atenuación en el corpus de comentarios de prensa frente al corpus de tuits. Investigaciones de corte comunicativo o sociológico sobre el perfil de los usuarios de cada plataforma y su percepción del ámbito comunicativo en el que interaccionan podrían enriquecer el estudio de la atenuación en estas interacciones. Ahora bien, junto a esta constatación, el análisis llevado a cabo también pone de relieve algunas tendencias.

La primera pregunta de investigación se centraba en los contextos comunicativos de aparición de las estrategias de atenuación. A este respecto, el presente análisis pone de manifiesto que los fenómenos de atenuación se manifiestan sobre todo en contextos interaccionales, ya que aparecen principalmente en intervenciones reactivas a lo dicho por uno o varios interlocutor(es) específico(s). Este resultado enfatiza el aspecto social de la atenuación según el cual esta estrategia lingüística busca lograr el acercamiento social al otro (Briz, 2012), en la medida en que aparece con más frecuencia en intervenciones en las que el hablante concibe un destinatario específico cuya imagen podría verse afectada.

Nuestra segunda pregunta de investigación se interesaba por las temáticas tratadas en los actos de habla atenuados. De forma general, no observamos diferencias importantes en la aparición de la atenuación según la temática tratada, la fiesta o la sociedad. Esta observación nos lleva a la conclusión de que las tácticas de atenuación no aparecen necesariamente en actos que tratan del aspecto polémico de la fiesta y parece corroborar el estudio de Moya Muñoz y Carrió-Pastor (2018) según el cual la presencia de la atenuación no varía significativamente según la temática. La investigación en la que se enmarca este artículo mostrará si estos resultados se comprueban con otras fiestas tradicionales.

No obstante, el presente análisis también resalta fenómenos de atenuación en actos de habla que consisten en una crítica o un ataque, sobre todo cuando se dirigen a un internauta específico, lo cual responde al deseo de atenuar el efecto negativo en el interlocutor. Además, observamos que los fenómenos de críticas atenuadas superan los actos de habla atenuados sobre la fiesta y la sociedad en el corpus de comentarios de prensa. Esta observación pone de manifiesto, por un lado, la presencia de la atenuación en contextos de interacción caracterizados por posibles efectos negativos para un interlocutor y, por otro lado, el carácter poco atenuado de la expresión de posturas en la sociedad española en un género cuya principal característica es esa. Así, un análisis profundizado de estos fenómenos con un corpus más amplio podría enriquecer la investigación sobre la violencia verbal en línea aportándole una nueva perspectiva y 
ahondando en el estudio de los comportamientos comunicativos en los dos géneros digitales aquí estudiados.

La tercera pregunta formulada se centraba en los tipos de actividad de imagen realizados por la atenuación, a través del análisis de su función. Esta estrategia pragmática aparece en las interacciones analizadas sobre todo con función preventiva y, en menor medida, pero también con mucha frecuencia, con función autoprotectora en ambas plataformas. Estos resultados divergen de los obtenidos por Pano Alamán (2018), quien observó una mayor presencia de casos de autoprotección en los comentarios de prensa sobre temas políticos, especialmente cuando el tema se considera delicado. Una posible explicación de esta diferencia podría relacionarse con las imágenes implicadas y los tipos de intervenciones. Miembros de los grupos sociales atañidos por las controversias en torno a la fiesta podrían formar parte del público lector y comentarista en las discusiones, lo que implicaría ciertas imágenes y, por consiguiente, determinadas estrategias de protección de estas.

Finalmente, nuestro último objetivo era identificar los procedimientos lingüísticos que se utilizan más para atenuar lo dicho. Al respecto, observamos que las estrategias lingüísticas más frecuentes son las que permiten suavizar la fuerza ilocutiva del acto de habla, principalmente el uso del condicional y de verbos cognitivos con valores modales. Este resultado corrobora las observaciones hechas en los estudios sobre la atenuación en comentarios de prensa en los que se destaca una predominancia de tácticas que permiten expresar duda o incertidumbre (Pano Alamán, 2018; Moya Muñoz y Carrió-Pastor, 2018).

También observamos en el corpus de Twitter varios usos de operadores modales y aún más de elementos propios del código escrito digital en contextos de prevención de la imagen del interlocutor. Cabría comprobar entonces con datos más numerosos si la presencia de estas tácticas de atenuación se restringe a las intervenciones en Twitter y, siendo ese el caso, preguntarse por qué aparecen en este tipo de intervención y no en los comentarios de prensa. Las características específicas de Twitter en cuanto a la breve extensión de los mensajes, al grado de formalidad de las intervenciones, así como la aceptación de usos del código escrito digital con función atenuante por parte de los usuarios podrían avanzar pistas.

En conclusión, el análisis de interacciones en comentarios a artículos de prensa y en Twitter sobre una fiesta tradicional controvertida por el tratamiento o la representación de ciertos grupos sociales permitió ampliar el campo de investigación sobre la atenuación. Por un lado, se analizaron intercambios sobre un tema nuevo, relacionado con tradiciones en España y reflejo de polémicas vigentes en la actualidad española. Por otro lado, se estudiaron intercambios en plataformas distintas que comparten el hecho de posibilitar expresarse sobre temas de actualidad, lo cual permitió formular algunas observaciones sobre los comportamientos comunicativos propios en ambas plataformas. 


\section{AGRADECIMIENTOS}

Agradecemos los valiosos comentarios de Barbara De Cock (Université catholique de Louvain) y de revisores anónimos sobre las versiones anteriores de este artículo.

\section{BIBLIOGRAFÍA}

AAKHUS, Mark y Marcin LEWINSKI (2017): "Advancing Polylogical Analysis of Large-Scale Argumentation: Disagreement Management in the Fracking Controversy», Argumentation, 1, 31, 179-207, https://doi.org/10.1007/s10503016-9403-9.

AlbELDA, Marta (2010): «¿Cómo se reconoce la atenuación? Una aproximación metodológica basada en el español peninsular hablado", en Orletti, Franca y Laura Mariottini, eds., (Des)cortesía en español. Espacios teóricos y metodológicos para su estudio, Roma/Stockholm, Università Roma Tre/Stockholm University, 47-70 [en línea]: http://www.edice.org/descargas/4coloquioEDI CE.pdf. [Consulta: 23/03/2021]

AlbELDA, Marta y María Estellés (2021): «Mitigation revisited. An operative and integrated definition of the pragmatic concept, its strategic values, and its linguistic expression", Journal of Pragmatics, 183, 71-86.

AlbeldA, Marta y Antonio BrIZ (2020): «Atenuación e intensificación», en ESCANDELL VIDAL, M. Victoria, Aoife Kathleen AHERn y José AmENós Pons, eds., Pragmática, Madrid, AKAL, 567590.

Albelda, Marta y Ana María Cestero (2011): «De nuevo, sobre los procedimientos de atenuación lingüística», Español Actual, 96, 940.

Albelda, Marta, Antonio Briz, Ana María Cestero, Dorota KotwICA y Cristina VILLALBA (2014): «Ficha metodológica para el análisis pragmático de la atenuación en corpus discursivos del español. (ES.POR.ATENUACIÓN)» Oralia, 17, 7-62.

AULIT, Laetitia (manuscrito): «Methodological proposal for the study of mitigation in conflictual online interactions".

Bravo, Diana (1999): "¿lmagen "positiva” vs. Imagen "negativa"?: Pragmática socio-cultural y componentes del face», Oralia, 2,155-184.

BRIZ, Antonio (2005): «Eficacia, imagen social e imagen de cortesía. Naturaleza de la estrategia atenuadora en la conversación cotidiana española», en Bravo, Diana, eds., Estudios de la (des)cortesía en español, Stockholm/Buenos Aires, EDICE/Editorial Dunken, 53-91.
BRIZ, Antonio (2012): «La (no)atenuación y la (des)cortesía, lo lingüístico y lo social: ¿son pareja?», en ESCAMILLA MORALES, Julio y Grandfield HENRY VEGA, eds, Miradas multidisciplinares a los fenómenos de cortesía y descortesía en el mundo hispánico, Barranquilla/Stockholm, Stockholm universitet/Universidad del Atlántico, 33-75.

Brown, Penelope y Stephen C. LEVINSON (1978): "Universals in language usage: Politeness phenomena", en Goody, Esther N., eds., Questions and politeness. Strategies in social interaction, Cambridge, Cambridge University Press, 56-311.

Brown, Penelope y Stephen C. LeVInson (1987): «Politeness: Some universals in language usage», Cambridge, Cambridge University Press.

CAFFI, Claudia (1999): «On mitigation», Journal of Pragmatics, 31, 881-909, https://doi.org/10.1016/S03782166(98)00098-8.

CHODOROWSKA-PILCH, Marianna (2017): «Atenuación pragmática: el caso de las condicionales», Normas, 1, 7, 97-106, http://dx.doi.org/10.7203/Normas.7.10426.

DE COCK, Barbara, Aurélie MARSILY, Andrea PizarRo Pedraza y Marie Rasson (2018): "¿Quién atenúa y cuándo en español? La atenuación en función del género discursivo", Spanish in Context, 2, 15, 305-324, https://doi.org/10.1075/sic.00016.dec.

DRESNER, Eli y Susan C. HERRING (2010): «Functions of the Non-Verbal in CMC: Emoticons and Illocutionary Force», Communication Theory, 3, 20, 249-268, https://doi.org/10.1111/j.14682885.2010.01362.x.

FigueRAS BATE, Carolina (2014): «Pragmática de la puntuación y nuevas tecnologías», Normas, 4, 135-160, https://doi.org/10.7203/Normas.4.4691.

GoFFMAN, Erving (1967): Interaction Ritual: Essays in Face-to-Face Behavior, Chicago, Aldine.

GOFFMAN, Erving (1981): Forms of Talk, Philadelphia, University of Pennsylvania Press.

GONZÁlEZ GARCíA, Virginia y Amparo GaRCíA RAMÓN (2017): «Atenuación e intensificación: estrategias pragmáticas del rechazo en 
respuestas a invitaciones en redes sociales en línea», en AlBeLDA, Marta y Wiltrud MiHATSCH, eds., Atenuación e intensificación en géneros discursivos, Madrid/Frankfur am Main, Iberoamericana/Vervuert, 187-204.

KotWICA, Dorota, ed. (2017): Lenguaje vago y atenuación II, Normas, 2, 7.

LAKOFF, George (1973): «Hedges: A Study in Meaning Criteria and the Logic of Fuzzy Concepts», Journal of Philosophical Logic, 4, 2 , 458-508.

Mancera Rueda, Ana (2015): «Los estudios sobre (des)cortesía y actividades de imagen en las redes sociales: notas para un estado de la cuestión», Textos en Proceso, 1, 1, 50-70, http://dx.doi.org/10.17710/tep.2015.1.1.3ma $\underline{\text { n. }}$.

MOYA MUÑOZ, Patricio y María Luisa CARRIÓ-PASTOR (2018): «La atenuación en los comentarios sobre las noticias digitales en periódicos de España y Chile», Onomázein, 40, 56-76, http://dx.doi.org/10.7764/onomazein.40.04.

NAETS, Hubert (2018): «Techniques de collecte et d'archivage des tweets: Partage de pratiques et d'outils", en Françols, Aurore, Anne ROEKENS, Véronique FILLIEUX y Caroline DERAuw, eds., Pérenniser l'éphémère. Archivage et médias sociaux, Louvain-laNeuve, Academia Eds, 215-237.

NoBLíA, Valentina (2018): «La interacción laboral en redes sociales móviles. El uso de los modos como estrategia de atenuación", Círculo de lingüística aplicada a la comunicación, 73, 77102, https://doi.org/10.5209/CLAC.59060.

PAGE, Ruth (2014): «Saying 'sorry': Corporate apologies posted on Twitter», Journal of Pragmatics, 62, 30-45, https://doi.org/10.1016/i.pragma.2013.12.003
PANO ALAMÁN, Ana (2018): «Opinión y atenuación en los comentarios de la prensa digital española», Círculo de lingüística aplicada a la comunicación, 73, 103-124, https://doi.org/10.5209/CLAC.59061.

SCHNEIDER, Stefan (2010): «Mitigation», en LOCHER, Miriam A. y Sage L. GraHAM, eds., Interpersonal pragmatics, Berlin, De Gruyter, 253-269, https://doi.org/10.1515/9783110214338.2.25 3.

SCHNEIDER, Stefan (2017): «Las dimensiones de la intensificación y de la atenuación», en ALBELDA, Marta y Wiltrud MiHATSCH, eds., Atenuación e intensificación en géneros discursivos, Madrid/Frankfur am Main, Iberoamericana/Nervuert, 23-42.

SEARLE, John R. (1969): Actos de habla. Ensayo de filosofía del lenguaje, Madrid, Cátedra.

SOLER BONAFONT, M. Amparo (2016): «Implicaciones pragmáticas del sujeto en el verbo "creo"”, en BENITO RuIz, Ángela, Pedro Pablo Espino RodríGuez y Bruno Revenga SaIz, eds., Nuevas investigaciones lingüísticas. $X X X$ Congreso Internacional de la Asociación de Jóvenes Lingüísticas, Santander, Editorial de la Universidad de Cantabria, 373-389.

VILLALBA IBÁÑEZ, Cristina, ed. (2017): Lenguaje vago y atenuación I, Normas, 1, 7.

VILLALBA IBÁÑEZ, Cristina (2018): «Atenuación: algunas claves metodológicas para su análisis», Normas, 1, 8, 306-316, https://doi.org/10.7203/Normas.v8i1.13277.

Yus, Francisco (2014): "Not all emoticons are created equal», Linguagem em (Dis)curso, 3, 14, 511-529, http://dx.doi.org/10.1590/19824017-140304-0414. 